\title{
Effective Communication between Nurses and Doctors: Barriers as Perceived by Nurses
}

\section{Amudha P, Hamidah H, Annamma $\mathrm{K}^{*}$ and Ananth N}

School of Nursing, KPJ Healthcare University College, Lot PT 17010, Persiaran Seriemas, Kota Seriemas, Nilai, Negeri Sembilan Darul Khusus, Malaysia

*Corresponding author: Annamma Kunjukunju, School of Nursing, KPJ Healthcare University College, Lot PT 17010, Persiaran Seriemas, Kota Seriemas, Nilai, Negeri Sembilan Darul Khusus, Malaysia, Tel: 606-7942131/2632; Fax:606-7942662; E-mail: ann@kpjuc.edu.my

Received date: Apr 22, 2018; Accepted date: Jun 05, 2018; Published date: Jun 12, 2018

Copyright: ( 2018 Amudha P, et al. This is an open-access article distributed under the terms of the Creative Commons Attribution License, which permits unrestricted use, distribution, and reproduction in any medium, provided the original author and source are credited.

\begin{abstract}
Effective communication among healthcare providers is the key driver for the success of the healthcare system. All the decisions related to patient care depend on effective communication among healthcare providers. Communication and teamwork are the backbones of the organization and helps to safeguard patients' safety. The study aimed to identify the contributing factors to the communication gap between doctors and nurses at selected private hospitals in Malaysia. The study used a qualitative method with an explorative and descriptive design to elicit the experience of 24 staff nurses from six private hospitals in Kuala Lumpur, Malaysia. A semi-structured interview was conducted to collect the data. The data were analysed using Colaizzi's method for thematic data analysis. The findings of the study suggested three categories as the factors to be responsible for the nurse-physician communication gap as perceived by nurses. The three categories are nurses work readiness; work environment and physician attributes. The respondents also suggested measures to overcome the communication barrier among nurses and physicians. In conclusion, a healthy nurse-physician communication is a vital factor in determining patient safety and quality of care.
\end{abstract}

Keywords: Communication; Qualitative research; Nurse-doctor communication; Barriers

\section{Introduction}

The Joint Commission on Accreditation (JCA) set up the Patient Safety Goals (PSG) in 2003 to address the significant patient safety issues. In the year 2004, Malaysian Health Ministry established National the Patient Safety Goals (NPSG) to address patient safety concerns in Malaysian healthcare context. The NPSG goal number two is to "Improve Effective Communication." The standard requires the organization to develop, establish and implement a structured, standardized tool to improve communication skills among healthcare providers.

In healthcare organizations, the type of nurse-physician relationship and effectiveness of internal communication is one of the critical elements in deciding the quality of patient care. Moreover, effective communication helps to make collaborative decisions toward patientcentered care and promote positive outcomes [1]. Overall, the quality of patient care in healthcare organizations are highly related to effective communication among physicians and staff nurses. The types of nurse -physician relationship and also determines patient safety.

Schmalenberg and Kramer in their research article reported findings synthesized from six research articles on nurse-physician communication. This article published story of 20,000 staff nurses how they perceive, assess and develop high-quality relationships with physicians in hospitals to improve patient care. The study reported different types of nurse-physician relationships. The types of nursephysician relationship as specified by the researchers include collegial relationships, collaborative relationships; student-teacher relationships; friendly stranger relationships and hostile relationships. The features of collegial relationship include equal trust; power and respect characterize a collegial relationship. Nurses and physicians refer to each other as peers in their relationship. Doctors are excellent and value the opinion of staff nurses. Then again, in the collaborative relationships, there is mutual trust among nurses and doctors. In the collaborative nurse-physician relationships is based on the shared trust; power; respect and cooperation are based on mutuality than equality. In collaborative relationships, nurse and physicians listened to one another plan of care but "doctors are still on top" as reported by the researchers, the best nurse-physician relationships are collegial relationships and collaborative relationships. Conversely, in the student teacher nurse-physician relationships, either the physician or the nurse can be a teacher. With residents or attending physicians who are dealing with comorbidities beyond their specialty, the nurses may take teaching or guiding role. In friendly stranger nurse-physician relationship there is a formal exchange of information and a neutral feeling tone of rapport. The worse nurse-physician relationships are hostile. Moreover, these type of relationship characterized by anger; verbal abuse real or implied threats and resignations [2].

However, throughout the entire history nurses and physicians have shared a complicated relationship [3]. The nurse-physician relationship is influenced by the power of authority, social status, gender and other perspectives. Tensions, conflicts as well as misunderstandings caused by the difference in opinions and interest among nurses and physicians upset the relationship. The lack of good relationship can interfere with effective interdisciplinary communication and collaboration. Improving the patient safety requires addressing the current hierarchical professional relationship inherent in healthcare delivery [4]. It is highly relevant that efforts are taken to determine the type of nurse-physician relationship in the local context.

The purpose of the study was to identify the existing type of nursephysician relationship and factors contributing to the current nurse- 
physician relationships as perceived by the Registered nurses in a private healthcare setting in Malaysia.

\section{Background of the study}

The study has been conducted in six selected private hospitals in Malaysia. The six private hospitals are under one flagship of KPJ healthcare organization. The organization has a total of 27 hospitals across Malaysia, Indonesia, and Bangladesh. There are more than 500 specialists and more than 3000 nurses employed across the network of the KPJ Healthcare hospitals. All the hospitals are tertiary level specialist hospitals providing a wide range of specialist medical services across all medical disciplines. All the registered nurses possess Diploma in nursing qualification which is the basic nursing qualification for a Registered nurse in Malaysia. However, many of the Registered nurses also have Post basic qualification and are specialist nurses. They had been trained mainly by KPJ Healthcare University College (Nilai) or KPJ healthcare college (Johor Baharu). However, the organizations also have a good number of nurses trained in other School of Nursing across Malaysia. All the nurses working for the organization and providing direct patient care are locals. The specialist had been trained in varieties of settings across the globe.

From the researcher's personal experience as a nurse educator, there had been criticisms about nurse's inability to effectively communicate with the physicians. It is precisely relevant to junior nurses with minimal clinical experience. The poor communication results when nurses are unsure of patient details and lead to poor case reporting during the ward rounds as well as telephone case reporting skills. Sometimes there is poor comprehension of medical terminologies commonly used in the medical discipline among nurses especially the junior nurses with less than two years of experience in the same unit. The poor clinical communication affects respect, trust and confidence among nurses and physicians.

\section{Aim}

This study aimed to identify the factors influencing communication gap between nurses \& medical doctors working in selected private hospitals in Malaysia.

\section{Research questions}

i. What are the factors affecting the communication gap between nurses and physicians as perceived by nurses?

ii. What strategies could be used to improve communication among nurse and physicians?

\section{Methods and Methodology}

\section{Research design}

The study used a qualitative method with descriptive, explorative design to explore factors affecting the nurse-physician relationship. A semi-structured interview was conducted with each of the respondents until saturation of data was obtained. All the interviews were tape recorded with the consent of the respondents.

\section{Study settings}

The study settings involved six private hospitals in Malaysia. All the three hospitals are within Klang Valley in Kuala Lumpur and are under the management of KPJ Healthcare organization. The staff nurses employed in general medical-surgical units were chosen as the study respondents.

\section{Sampling technique}

A convenient sampling technique was used for data collection. After obtaining the permission from the Chief Nursing officers of the respective hospitals the researcher personally contacted the unit managers from the medical, surgical units. The unit manager referred the researcher to few staff nurses who were the potential respondents. The researcher then personally contacted the prospective respondents and after initial self-introduction, the researcher briefly introduced the study topic and appointment was made for the interview. Interviews were conducted in a comfortable staff room within the ward of each of the respondents. Explanatory consent was obtained before commencement of the interview with each of the respondents.

The number of samples was decided based on data saturation. A total of 24 registered nurses were the respondents for the study.

\section{Inclusion criteria}

The staff nurses who had been willing to give the explanatory consent were included as the respondents for the study.

\section{Exclusion criteria}

The staff nurses who were not willing to participate and sign explanatory statement were excluded for the study.

\section{Data collection method}

The study used semi-structured questions for data collection. The semi-structured interview was conducted in the hospital premises. Prior to the data collection, the researcher had obtained permission from the Chief Nursing Officer (CNO) of the respective hospitals. The CNO's referred the researcher to the unit managers of the medicalsurgical units. With the permission and referral of the unit manager the researcher personally contacted the staff nurses and introduced self and an appointment was obtained to have the semi-structured interview. Before commencement of the interview the topic and purpose of the study was reinstated to the respondents and obtained the written consent using explanatory statement. Then, the researcher collected demographic data's followed by a semi-structured interview was conducted regarding communication gaps between nurses and doctors. All the interviews were tape recorded with consent of the respondents.

\section{Data analysis}

The interviews were tape-recorded and verbatim were transcribed. The data analysis used manual qualitative analysis from the field notes and the verbatim. The transcript was read and reread independently by the researchers to obtain the themes related to the nurse-physician communication gap.

\section{Ethical clearance}

This study has received the Ethical Clearance/approval from the Ethical Committee of KPJ Healthcare University College. An explanatory statement was given and consent was obtained from the participants before the data collection. The data in the tape recorder 
Page 3 of 6

was transferred to the laptop and was password protected to be accessible only to the research team. The transcribed verbatim was stored in a dedicated box to safeguard the confidentiality of the data.

\section{Results}

\section{Factors contributing to communication gaps between nurses \& doctors}

From the qualitative data analysis using Colaizzi's method of qualitative data analysis, two categories with 12 themes emerged from the data (Tables 1 and 2). The three categories emerged from the data are: nurses work readiness; work environment and physician's attributes. Table 3 reports the categories and themes identified across the studies, along with example quotes from the respondents.

Nurse's work readiness: The preparedness of the staff nurses were one of the factors impacting the nurse-physician communication skills.

\begin{tabular}{|c|c|c|}
\hline Age in yrs. & Number (n) & Table 2: Staff nurses years of clinical experience in nursing. \\
\hline Categories & Themes & Examples \\
\hline \multirow[t]{4}{*}{ Nurse's work readiness } & $\begin{array}{l}\text { Lack of knowledge in the } \\
\text { specialty }\end{array}$ & $\begin{array}{l}\text { "Sometimes we are unable to answer doctor's questions and the doctors scold us. So we are scared and } \\
\text { next time we avoid those consultants' rounds. We expect them to teach us not scold us. We want to } \\
\text { learn." }\end{array}$ \\
\hline & $\begin{array}{l}\text { Lack of competency of new } \\
\text { graduate nurses }\end{array}$ & "At times we don't have skill in performing certain procedures". \\
\hline & Time management & $\begin{array}{l}\text { "We expect the consultants to understand our situation. We need to entertain patients, relatives and } \\
\text { everyone around us verbally and over the phone. We are answerable to everyone. If something goes } \\
\text { wrong, they get frustrated". }\end{array}$ \\
\hline & $\begin{array}{l}\text { Theoretical versus practical } \\
\text { training }\end{array}$ & $\begin{array}{l}\text { "For example procedure like dressing uses many solutions and materials. Each consultant has their } \\
\text { preference too College only teach basic dressing procedure". }\end{array}$ \\
\hline \multirow[t]{3}{*}{ Work environment } & Non -nursing job & "Insurance and billing take way lot of our time. Moreover, an admission itself has a lot of clerking job". \\
\hline & $\begin{array}{l}\text { Perception of being } \\
\text { appreciated }\end{array}$ & $\begin{array}{l}\text { "Even with all our sacrifices, nobody cares for us or our wellbeing. Sometimes we are sick. But because } \\
\text { of staff shortage still, need to work." }\end{array}$ \\
\hline & Staff shortage & "We need more care assistants. That can ease our burden." \\
\hline \multirow[t]{4}{*}{ Physicians attributes } & Power of authority & "Some doctors are bossy. Do not want to listen to any reason from us". \\
\hline & $\begin{array}{l}\text { Personality traits of } \\
\text { physicians }\end{array}$ & "One of the respondents exclaimed, "Some consultants are very loud, fast; some slow and soft." \\
\hline & Mood variations & $\begin{array}{l}\text { "Some consultant deal with us according to their stress and mood but not all consultants. Some are very } \\
\text { nice." }\end{array}$ \\
\hline & Handwriting & "Especially medication orders. We are worried about making mistakes." \\
\hline
\end{tabular}

Table 3: Factor related to poor nurse-physician communication.

Lack of knowledge in the speciality areas: Sixty-three percent of the staff nurses felt that they need to improve their knowledge in the related discipline. Registered nurses stated that sometimes they do not understand what the consultants communicate using medical terminologies. The knowledge deficit is especially real for new graduate nurses in the early stage of the professional role. For example, the information related to details of the drugs like IV Immunoglobulin and certain medical terms like 'Kawasaki disease'. Some even may hesitate to attend rounds with the same physicians and try to avoid the physician subsequently. All the respondents mentioned that having

\begin{tabular}{|l|l|l|}
\hline $21-30$ & 16 & 66 \\
\hline $31-40$ & 3 & 13 \\
\hline $41-50$ & 3 & 13 \\
\hline $51-60$ & 2 & 8 \\
\hline
\end{tabular}

Table 1: Age of the respondents (Staff nurses).

\begin{tabular}{|l|l|l|}
\hline Years of experience & Number & $\%$ \\
\hline$<1$ year & 8 & 33 \\
\hline $1-5 \mathrm{yr}$ & 4 & 17 \\
\hline $6-10 \mathrm{yr}$ & 5 & 21 \\
\hline$>10 \mathrm{yr}$ & 7 & 29 \\
\hline
\end{tabular}

Table 2: Staff nurses years of clinical experience in nursing. 
But they don't want to teach". Another respondent "Sometimes the consultants ask more detail about drug, I do not know. Then I avoid even go for rounds, or I attend along with senior until I know the expectation of the consultants".

Theoretical versus practical training: The student experience of theoretical learning with work placements differed significantly from working as an RN. Three (12\%) of the staff nurses responded: "What we learn (the basic procedures) in college is performed differently in a hospital setting" "For example surgical dressing". In College they teach simple surgical dressing. But in the clinical they use a variety of dressing materials and solutions. Sometimes we don't know what the consultant prefers. Some consultants are very good. But few may get angry and shout at us. Moreover, different doctors do the identical procedure in a different style. All these cause a lot of confusion for us in the ward".

\section{Work environment}

Staff shortage: Thirteen (54\%) nurses retorted that shortage of staff nurses in one of the major issues. Nurses stated that they need to do a double shift when some of the staffs go on Medical leave. Adapting to double shift work was acknowledged as a difficult part of the nursing role. Tiredness associated with this lifestyle change had negative consequences for nurses and their patient's wellbeing and quality of care. It is very exhausting. Sometimes we need to sacrifice even our meal time to meet the patients' demands. The shortage of staff can affect standard of nursing care from the physician point of view.

Non-nursing work: One of the respondents answered: "We do a lot of non-nursing jobs like insurance clearance. If paperwork is delayed patients and doctor will blame us". Sometimes the patients get admitted in the morning and get discharged evening. The process of settling bill may take few hours the patients will demand immediate settlement and if not settled immediately both the respective consultant and the patients will get frustrated with us. They don't understand our situation" Even for admission; we need to do a lot of clerical work. By the time we could finish the paper works, next patient will be in a queue for admission. "The delay cause frustration and we will be the victims even though we are not handing billing"

Time management of nurses: Management of time and related stress also were indirect factors respondents perceived to be contributing to the quality of work and hence affects effective communication among nurses and physicians. Nine (38\%) of the respondents stated "suddenly patient queue up admission, sending another patient to OT, answering call bell, attending doctors rounds and so on add stress. Sometimes we are not able to follow the doctors for rounds due to time constraints".

Perception of being appreciated: Respondents shared the hard work and sacrifices they make resulted in frustration when they felt their hard work was not appreciated by the physicians or the management of the organization. Sometimes we are too busy with ward work and unable to attend rounds. That may make consultant angry. They don't understand our situation. One of the respondent stated "Sometime we even sacrifice our time for meal breaks to care for patients especially when the workload is high due to staff shortage. But most of the times our hardships are not appreciated and may even get blamed for any unintended shortcomings".

\section{Attributes of physicians}

Personality traits of doctors: "Some consultants are very strict. If I don't know something, they don't want us to follow them for rounds next time. We feel upset. Actually if we don't know something we expect them to teach us not scold us". Five (21\%) of the staff nurses answered that they felt scared to talk with doctors because some of the doctors can be very "rough" and speak loudly. Each doctor has their ways of doing the procedures as well. It is challenging to understand the differences in doctor's practices. Some doctors order (treatment) verbally and if we ask for verification may become agitated. Sometimes we are unable to attend rounds and they may order new medication new medication order but not inform us or at times verbal order of medication not written in the medication record"

Power of authority: The attitudes and behaviour of the physician was an important factor decided the effectiveness on nurse-physician communication. Eight (33\%) staff nurses answered that doctors show their power of authority on nurses. For example," sometimes doctors may order medicine that is not available in the ward stock. By the time we get it from pharmacy, they may become annoyed and raise their voice." Sometimes we felt we are looked down when we give suggestion do not want to listen; but some appreciate \& accept".

Mood difference of doctors: Nine (38\%) of the respondents stated that doctors interaction with the staff nurses differs based on their mood. 'Sometimes the doctors may be upset with other units' staff or even patients." At one time Orthopaedic doctor prescribed steroid for pregnant mother and the obstetrician scolded us. Is it our mistake"? On the other hand, when they are in a good mood, they may also share a lot of information from conferences

Handwriting of doctors: Twelve (50\%) nurses stated that sometimes doctor's handwriting not is legible. "Sometimes we don't understand the medication. If we ask for clarification, they retaliate saying ask your senior. Sometimes the seniors are also not sure of the written prescription. Then we check with the Pharmacy or ask the consultant again". However, with the introduction of the computerized information system and prescriptions, this issue is becoming insignificant.

\section{Suggestions to improve the nurse-physician communication}

First and foremost nurses need to enhance knowledge of the related discipline and English language proficiency. Some respondents recommended that continuous professional development update to be taught by consultants, before consultants expect them to perform. Respondents hope that the consultants use opportunity like ward rounds for constructive teaching than scolding the nurses for their ignorance.

To help in adaptation process of newly hired staff respondents recommended having proper preceptor system. Most of the times preceptors are in a different shift that of the preceptee which doesn't help the preceptee. Newly hired staff also needs to learn preference of each consultant as quickly as possible. The respondents also acclaimed that the theory versus practical gap can be reduced with a more effective mentor-mentee system and hands-o n during student period. They suggested increasing the workforce to overcome work force shortage.

Nurses preferred not to be transferred to other wards, because of difficulty to adapt to the new ward. Doctors also must understand our situation when we are short of staff. Respondents recommended 
having more care assistant's help to reduce non-nursing work of RN They also recommended increasing $\mathrm{RN}$ work force to overcome $\mathrm{RN}$ shortage.

Doctors need to understand nurse's workload and react accordingly. More collaborative and communicative relationship than authoritarian relationship will help in better communication. Doctors need to write the order explicitly to carry out the order without any error. Talk nicely; teach more than complain or look down; unless an emergency, need to wait. Consultants need to understand nurse's situation of the ward and management of the organization need to appreciate the hard work of the 'unsung heroes'.

\section{Discussion}

The nurse-physician relationship set the tone for unit culture and hence all measures need to be taken to develop a collegial and collaborative relationship. One of the most important determinants of patient safety and quality of care offered by the hospitals will depend upon the nurse-physician relationship of the individual units and unit culture.

One of the primary reasons for the poor nurse-physician communication gap is the knowledge deficit of nurses related to the specialty discipline. Few other studies also reported similar findings of [5-7]. One of the ways to address the lack of knowledge can be the use of structured transition programs $[7,8]$.

. The diploma in nursing training only can prepare generalist nurses. Specialized training is needed as on the job training or continuing professional development to work confidently and safely in specialty clinical areas. Moreover, evaluation of knowledge competency should be done periodically to enhance their knowledge $[9,10]$.

Physicians still adopt authoritarian attitudes and sometimes nurse's opinions are not heard. The findings of the study are in consistency with conclusions of Tabak et al. in which nurses were apprehensive about challenging the physicians' greater power \& authority, feeling intimidated by the physicians' prestige \& authority [11].

There is a social and professional view that nurses are the second rate to doctors. However, there is a vast difference between the two professions 3. Professional responsibility of nurses and doctors differs. However, both doctors and nurses need to function as a pair of gloves to achieve the optimum patient care goal. Doctor's focus to cure the patient where else nurses strives to provide holistic care for the patients. Both the professions are mutually exclusive and one can't successfully function without effective communication and collaboration with the other. Effective communication is the key to have a collaborative relationship with the healthcare providers.

Another factor is unfriendly personality traits of doctors and power of authority. The findings are similar in few studies [2-4]. Training on the importance of good nurse-physician interdisciplinary relationship can enhance relationship issues and to develop healthy unit culture [2].

Shortage of nurses had been adding stress to the working environment. The finding is consistent with other study findings $[10,12,13]$.

Organisations need to have staff retention strategies to address the issue of the work force $[10,13,14]$.

Illegible Doctors handwriting is one of the reasons for unintentional mistakes like medication errors [15-17]. Legible handwriting avoids misunderstanding reduce medical error and improve patient safety. However, with the implementation of the electronic health record, this issue should become obsolete in the future [18]. According to the research, when nurses personally hear the physician's plan of care, the potential for misunderstanding decreases, which in turn decreases the risk of error. Most of the time nurses feel too difficult to understand the doctor's handwriting. Sometimes nurses scared to clarify their doubts with doctors who can result in miscommunication and error in treatment [19].

Lack of understanding about the professional role of nurses is one area that research has identified and attention to improving collaborative relationships. Fortunately, more evidence is suggesting that the relationship between nurses and physicians is slowly becoming collegial or collaborative. Strategies can, therefore, can assist to improve relations between nurses and physicians [20].

However, traditional gender differences still exist. Men, who are more present in the medicinal field, prefer precise, quick, fact-based communication. Women, who are more present in the nursing field, prefer in-depth discussion style to understand the reasoning behind specific situations. This disparity, though improving as gender gaps decrease, may contribute to failures in communication [21].

Lancaster et al. in their study reported the need for excellent interdisciplinary communication; collaboration; education and training among nurses and physicians to support positive patient care outcomes 4 .

The relationship between nurses and physicians has a long history, from a time when physicians were dominant over subservient nurses to now where there is more collaboration between the two professions. Currently, collaborative relationships between nurses and physicians are becoming the norm. A collaborative relationship is significant between nurses and doctors to maintain efficient care [2].

Nurses need appreciation for their contribution to the organization in achieving organization goal [22]. The unit culture and organizational culture need to monitor and pleasant working environment need to be encouraged to help job satisfaction of nurses and to enhance staff retention. Nurses in magnet hospitals report a higher quality of nurse-physician relationships. In magnet hospitals, $80 \%$ of the nurse-physician relationship is collegial and collaborative [2]. The study findings from registered nurses interview reported 12 areas of the nurse-physician relationship need improvement in the study settings. Multiple factors are contributing to the weak nursephysician relationship in the study settings.

Work environments have many properties that may affect both physical and psychological well-being of the workers. Communication is a vital tool that health care professionals must use to elicit cooperation among individuals in the delivery of the healthcare services. It is an integral part of socialization \& imperative in establishing healthy relationships in the health community. The factors related to poor communication between both the professionals can be addressed constructively to improve unit culture and organizational culture.

\section{Limitations of the Study}

Fear of reprisals might have been real or perceived stressors for the respondents. In this study fear of retaliation had been addressed by taking every effort to maintain confidentiality and by making participants as comfortable as possible. No names or any identification 
was included in any notes or reports. The data collected were kept in a database on a private -password protected laptop computer. Another limitation of the study is small sample size. The study involved different study settings and as such, it was difficult to get an appointment with staff nurses due to different shifts and responsibilities.

\section{Recommendations}

The study can be conducted on a large sample to improve generalizations of study findings. The research also can be done using validated questionnaire survey on nurse-physician relationships to get more sizeable samples. A survey method may also avoid the fear of reprisal due to anonymity.

\section{Conclusion}

In this study, the nurse-physician communication gap related factors identified are grouped into three categories namely, nurses work readiness; work environment and physician attributes. From the study findings, the researcher concludes that there is a need to impart knowledge about excellent communication skills among nurses and to identify strategies to enhance effective communication between doctors and nurses. The nurses and doctors have a vital role in creating a healthy communication and collaboration to enhance excellent patient outcomes.

\section{References}

1. Sheldon LK, Hilaire DM (2015) Development of communication skills in healthcare: Perspectives of new graduates of undergraduate nursing education. J Nurs Educ Pract 5:7.

2. Schmalenberg C, Kramer M (2009) Nurse-physician relationships in hospitals: 20000 nurses tell their story. Crit Care Nurse 29: 74-83.

3. McKay KA, Narasimhan S (2012) Bridging the gap between doctors and nurses. J Nurs Educ Pract 2: 10-14.

4. Lancaster G, Kolakowsky-Hayner S, Kovacich J, Greer-Williams N (2015) Interdisciplinary communication and collaboration among physicians, nurses, and unlicensed assistive personnel. J Nurs Scholarsh 47: 275-284.

5. Gough J, Johnson L, Waldron S, Tyler P, Donath S (2009) Clinical communication: Innovative education for graduate nurses in pediatrics. J Nurs Educ Pract 9: 209-14.

6. Kelley TF, Brandon D (2012) Development of an Observational Tool to Measure Nurses' Information Needs. NI2012: 11th International Congress on Nursing Informatics, 209-213.
7. Brown RA, Crookes PA (2016) What are the "necessary" skills for a newly graduating RN? The results of an Australian survey. BMC Nursing 15: 18 .

8. Mooney M (2007) Newly qualified Irish nurses' interpretation of their preparation and experiences of registration. J Clin Nurs 16: 1610-1617.

9. AL-Dossary R, Kitsantas P, Maddox PJ (2014) The impact of residency programs on new nurse graduates' clinical decision-making and leadership skills: A systematic review. Nurse Educ 34: 1024-1028.

10. Duclos-Miller PA (2011) Successful graduate nurse transition: Meeting the Challenge. Nurse Lead 9: 32-35.

11. Tabak N, Orit K (2007) Relationship between how nurses resolve their conflicts with doctors, their stress and job satisfaction. J Nurs Manag 15: 321-331.

12. Dapremont J, Lee S (2013) Partnering to educate: Dedicated education units. J Nurs Educ Pract 13: 335-337.

13. Elmers CR (2010) The role of preceptor and nurse leader in developing intensive care unit competency. Crit Care Nurs Q 33: 10-18.

14. Gardiner I, Sheen J (2016). Graduate nurse experiences of support: A review. Nurse Educ Today 40: 7-12.

15. Cloete L (2015) Reducing medication errors in nursing practice. Nurs Stand 29: 50-59.

16. Wallace LS, Keenum AJ, DeVoe JE, Bolon SK, Hansen JS, et al. (2014) Assessment of knowledge of pediatric nurses related with drug administration and preparation. Nurse Educ Today 34: 1.

17. Yang Z, Ng BY, Kankanhalli A, Luen Yip JW (2012) Workarounds in the use of IS in healthcare: A case study of an electronic medication administration system. Int J Hum Comput Stud 70: 43-65.

18. Maaskant JM, Smeulers M, Bosman D, Busink A, van Rijn-Bikker P, et al. (2015) The trigger tool as a method to measure harmful medication errors in children. J Patient Saf 14: 95-100.

19. Burns K (2011) Nurse-physician rounds: A collaborative approach to improving communication, efficiencies, and perception of care. Medsurg Nurs 20: 194-200.

20. Tang C, Chan S, Zhou W, Liaw S (2013) Collaboration between hospital physicians and nurses: An integrated literature review. Int Nurs Rev 60: 291-302.

21. Flicek CL (2012) Communication: A Dynamic between nurses and physicians. Medsurg Nurs 21: 385-386.

22. Ebert L, Hoffman K, Levett-Jones T, Gilligan C (2014) "They have no idea of what we do or what we know": Australian graduates' perceptions of working in a healthcare team. J Nurs Educ Pract 14: 544-550. 